\title{
Triple-Therapie aus einem Inhaler punktet in zwei großen Studien
}

\author{
Die COPD-Triple-Therapien aus einem Inhaler \\ stehen ante portas: In den Studien TRILOGY und \\ TRINITY hat die Fixkombination ICS/LABA/LAMA \\ gegenüber ICS/LABA bzw. LAMA allein Exazerbatio- \\ nen wirksamer verhindert und auch die Lungen- \\ funktion deutlicher gebessert.
}

Wenn eine chronisch obstruktive Lungenerkrankung (COPD) häufig exazerbiert, empfehlen die GOLD-Leitlinien zunächst eine Therapie mit einem LAMA oder eine Kombination ICS/ LABA. Beide Therapien verbessern in diesem Stadium die Symptomatik, die Lungenfunktion, und das Exazerbationsrisiko.

\section{TRILOGY-Studie testet ICS/LABA/LAMA versus ICS/LABA}

Aus diesem Grund wurden diese beiden Therapien als Vergleichspartner für die Triple-Therapie gewählt. Diese besteht aus der extrafeinen Formulierung von Beclomethason-Dipropionat $(100 \mu \mathrm{g})$, Formoterol-Fumarat $(6 \mu \mathrm{g})$ und Glycopyrronium-Bromid $(12 \mu \mathrm{g})$, kurz BDP/FF/GB, der Firma Chiesi. In der randomisierten doppelbinden TRILOGY-Studie, publiziert zum ERSKongress 2016 im Lancet, wurde sie gegen BDP/FF verglichen, der Unterschied bestand also im lang wirksamen Parasympatholytikum Glycopyrronium. Von beiden Medikamenten wurden zweimal täglich zwei Hübe per Dosieraerosol verabreicht.

Studienteilnehmer waren 1.368 Patienten mit schwerer COPD, deren $\mathrm{FEV}_{1}$ im Schnitt bei 36\% lag (1,1 Liter), von denen die Hälfte weiter rauchte, und die im Jahr vor der Studie im Schnitt 1,2 Exazerbationen erlitten haben.

\section{Bessere Lungenfunktion, weniger Exazerbationen, aber keine Beschwerdereduktion}

Es gab drei co-primäre Endpunkte, die nach 26 Wochen erhoben wurden. Zum einen die FEV ${ }_{1}$ vor der nächsten Dosis. Diese

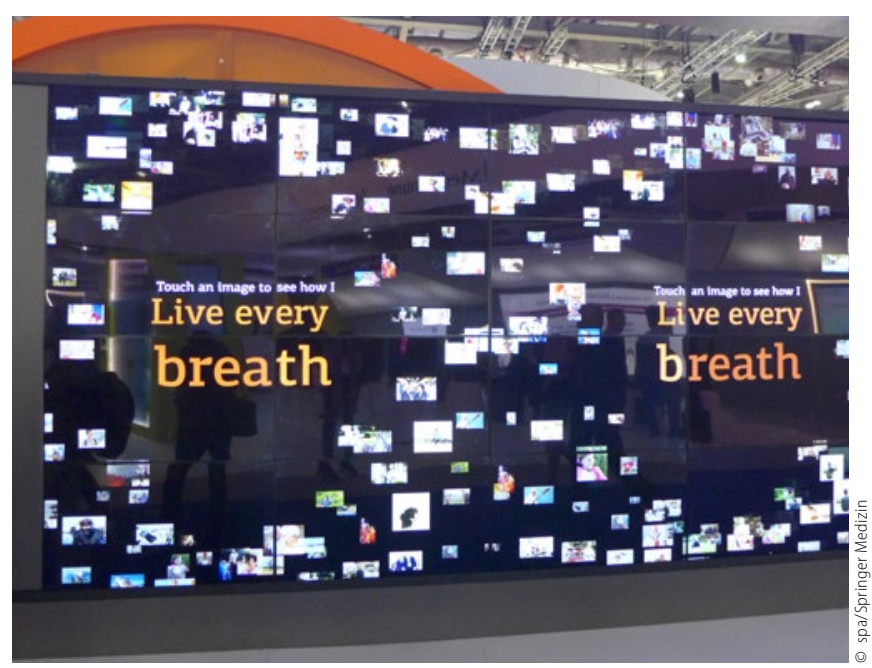

Die Bilderwand zeigte beim ERS-Kongress, dass das Leben auch noch mit einer Lungenerkrankung Spaß machen kann. verbesserte sich unter der Tripletherapie gegenüber der dualen Therapie um $81 \mathrm{ml}$, der Unterschied war signifikant. Zweiter Endpunkt war die $\mathrm{FEV}_{1}$ zwei Stunden nach der Applikation. Diese verbesserte sich unter drei Medikamenten im Schnitt um $117 \mathrm{ml}$ mehr als unter zwei Medikamenten, auch dies war signifikant. Dritter Endpunkt waren die Beschwerden, bemessen im TDI (Transition Dyspnea Index). Hier gab es keinen signifikanten Unterschied (1,71 vs. 1,5$)$.

Darüber hinaus reduzierte die Triple-Therapie das Risiko für mittelschwere und schwere Exazerbationen: Dieses war sehr niedrig und betrug auf ein Jahr hochgerechnet $0,41 \%$ unter Triple- und 0,53\% unter der dualen Therapie, was einer signifikanten 23\%igen Reduktion entspricht. Allerdings ist die Number Needed to Treat (NNT), um eine Exazerbation zu verhindern, sehr hoch.

\section{TRINITY-Studie testet ICS/LABA/LAMA versus LAMA}

Die beim ERS-Kongress in einer Hotline-Session vorgestellte TRINITY-Studie war ähnlich angelegt. 2.580 Patienten wurden in 2:2:1 Randomisierung auf die gleiche Tripletherapie BDP/FF/ GB eingestellt wie in der TRILOGY-Studie, auf eine Monotherapie mit Tiotropium, oder auf die freie Kombination DDP/FF plus Tiotropium aus zwei Inhalern. Auch in dieser Studie betrug die durchschnittliche $\mathrm{FEV}_{1}$ der Patienten 37\%, knapp 50\% der Patienten waren Raucher, im Vorjahr hatten sie im Schnitt 1,3 Exazerbationen erlitten. Primärer Endpunkt war hier jedoch die Exazerbationsrate nach 52 Wochen, sekundäre Endpunkte waren die $\mathrm{FEV}_{1}$ vor der Applikation.

Der primäre Endpunkt wurde erreicht: Patienten unter Tiotropium erlitten im Schnitt 0,57 Exazerbationen pro Jahr und unter den Tripletherapien 0,457 bzw. 0,452 pro Jahr. Die Unterschiede waren signifikant. Schwere Exazerbationen erlitten 0,098\% (Tiotropium) und 0,067\% bzw. 0,057\% (Tripletherapien). Aufgrund der geringen absoluten Unterschiede sind auch hier die NNTs, um eine Exazerbation zu verhindern, sehr hoch. Die morgendliche $\mathrm{FEV}_{1}$ besserte sich um $61 \mathrm{ml} \mathrm{mehr}$ als unter Tiotropium. Zwischen der Fixkombination und der freien Kombination der drei Medikamente gab es keine Unterschiede.

Vergleich mit dualer Bronchodilatation steht noch aus Die Studien* füllen eine Wissenslücke in der Therapie der COPD, schrieb der Liverpooler Pneumologe Prof. Peter Calverley in einem Lancet-Editorial. Allerdings beantworten sie nicht alle Fragen: Es muss noch untersucht werden, wo und ob die Dreifachkombination der dualen Bronchodilation mit LABA/ LAMA überlegen ist. Die WISDOM-Studie hatte diesbezüglich ein Fragezeichen gesetzt.

Dr. med. Dirk Einecke

\section{*Beide Studien wurden von der Firma Chiesi finanziert.}

Quellen: International Congress 2016 der European Respiratory Society ERS, London, 3.-7. September 2016; Singh D, et al.; Single inhaler triple therapy versus inhaled corticosteroid plus long-acting Beta-agonist therapy for chronic obstructive pulmonary disease (TRILOGY); a double-blind, parallel group, randomized controlled trial. Lancet 2016; 388: 963-73. 